PROCEEDINGS OF THE

AMERICAN MATHEMATICAL SOCIETY

Volume 127, Number 2, February 1999, Pages 583-587

S 0002-9939(99)04518-9

\title{
A NOTE ON THE RAMSEY PROPERTY
}

\author{
A. TSARPALIAS
}

(Communicated by Andreas R. Blass)

\begin{abstract}
An elementary setting of the classical Ramsey property is given, which leads to simple proofs of the relevant theorems of Galvin-Prikry and Silver.
\end{abstract}

\section{INTRODUCTION}

The setting of the classical Ramsey property in this note is as follows: Some facts for arbitrary subsets of the space $[\mathbf{N}]$ of infinite subsets of $\mathbf{N}$ are proved, which lead to easy proofs of the relevant theorems of Galvin-Prikry [4] and Silver [6]. Topological conditions are posed exactly at the points where they are necessary. Moreover, our proof of Silver's theorem is more direct and "constructive" than the previous ones.

The proof of Silver that every analytic subset of $[\mathbf{N}]$ is completely Ramsey uses deep metamathematical ideas involving forcing. A classical proof of Silver's theorem was given by Ellentuck [3] based on Marczewski's theorem that in a topological space the class of sets with the Baire property is closed under the Souslin operation. Also, Ellentuck obtained a topological characterization of the completely Ramsey sets, proving that a subset of $[\mathbf{N}]$ is completely Ramsey if and only if it has the Baire property in the topology introduced by him. This characterization played a substantial role in the subsequent development of qualitative Ramsey theory, see Carlson [1], Carlson-Simpson [2]. Finally a proof of Silver's theorem using Ellentuck's characterization of completely Ramsey sets and topological games is included in the book of A. Kechris [5, p. 155].

\section{Notation AND DEFinitions}

In this note $a, b, \ldots$ denote finite subsets of $\mathbf{N}$ and $A, B, L, \ldots$ denote infinite subsets of $\mathbf{N}$. We set $a<A$ if $\max (a)<\min (A)$. We also set

$$
[a, A]=\{a \cup L: L \subset A \& a<L\} .
$$

By $[\mathbf{N}]$ we denote the set of infinite subsets of $\mathbf{N}$ endowed with the topology of pointwise convergence. If $X \subset[\mathbf{N}]$ we set $X^{c}=[\mathbf{N}] \backslash X, \bar{X}$ denotes the closure of $X$ in $[\mathbf{N}]$, and $\widehat{X}$ denotes the set defined by

$$
L \in \widehat{X} \Leftrightarrow \forall[a, A](L \in[a, A] \Rightarrow[a, A] \cap X \neq \varnothing) .
$$

Received by the editors November 19, 1996 and, in revised form, May 16, 1997. 1991 Mathematics Subject Classification. Primary 04A20; Secondary 04A15.

(C)1999 American Mathematical Society 
The Baire space $\mathcal{N}$ is the space $\mathbf{N}^{\mathbf{N}}$ endowed with the product topology, considering $\mathbf{N}$ with the discrete topology, $s$ denotes finite sequences of $\mathbf{N}$, and $\sigma$ denotes infinite sequences of $\mathbf{N}$. For every $s$ we denote by $\mathcal{N}_{s}$ the set of members of $\mathcal{N}$ that extend $s$.

Definition. A subset $X$ of $[\mathbf{N}]$ is called completely Ramsey if for every $a, A$ there exists $B \subset A$ such that either $[a, B] \subset X$ or $[a, B] \subset X^{c}$.

\section{The SETting OF RESUlts}

The following lemma is essentially contained in Galvin-Prikry [4] with different statement and terminology. We include a proof because of the different statement since it plays a substantial role in the sequel.

3.1. Lemma. Let $X \subset[\mathbf{N}]$ be an arbitrary set. Then, for every a, $A$ there exists $B \subset A$ such that either

(i) $[a, B] \subset X$ or

(ii) $[a \cup b, L] \not \subset X$ for every $b \subset B$ and $L \subset B$.

Proof. We suppose that for every $B \subset A$, (i) fails and prove that (ii) holds for some $B \subset A$. The proof will be by induction. We assume without loss of generality that $a<A$.

Base. There exist $x_{1} \in A$ and $A_{2} \subset A$ such that for every $L \subset A_{2}$,

$$
\left[a \cup\left\{x_{1}\right\}, L\right] \not \subset X .
$$

Assume the contrary. We choose $y_{1} \in A$. Then the pair $\left(y_{1}, A\right)$ does not satisfy $(*)$, so there exists $B_{2} \subset A$ such that $\left[a \cup\left\{y_{1}\right\}, B_{2}\right] \subset X$. We choose $y_{2} \in B_{2}$ with $y_{2}>y_{1}$. Continuing in this way natural numbers and sets

$$
\begin{gathered}
y_{1}<y_{2}<\cdots, \\
A=B_{1} \supset B_{2} \supset \cdots
\end{gathered}
$$

are constructed with $y_{i} \in B_{i}$ and $\left[a \cup\left\{y_{i}\right\}, B_{i+1}\right] \subset X$. We set $B=\left\{y_{j}: j=\right.$ $1,2, \ldots\}$. Then $[a, B] \subset X$ which is absurd.

Induction step. Assume that natural numbers and sets

$$
x_{1}<x_{2}<\cdots<x_{k}
$$

$$
A=A_{1} \supset A_{2} \supset \cdots \supset A_{k} \supset A_{k+1}
$$

have been constructed such that for every $i \leq k, x_{i} \in A_{i}$ and for every $L \subset A_{i+1}$ and $b \subset\left\{x_{1}, \ldots, x_{i}\right\},[a \cup b, L] \not \subset X$. Then for every $b \subset\left\{x_{1}, \ldots, x_{k}\right\}$ and for every $L \subset A_{k+1},[a \cup b, L] \not \subset X$. Now we can find $x_{k+1}$ and $A_{k+2}$ extending (**), since otherwise repeating the previous argument we find $b \subset\left\{x_{1}, \ldots, x_{k}\right\}$ and $B \subset A_{k+1}$ such that $[a \cup b, B] \subset X$ which is absurd. Thus

$$
\begin{gathered}
x_{1}<x_{2}<\cdots, \\
A=A_{1} \supset A_{2} \supset \cdots
\end{gathered}
$$

are constructed such that every initial segment satisfies $(* *)$. We set $B=\left\{x_{j}: j=\right.$ $1,2, \ldots\}$. Then $B$ satisfies condition (ii).

Condition (ii) says exactly that $[a, B] \subset \widehat{X^{c}}$. Hence as $X \subset \widehat{X} \subset \bar{X}$ we have the following.

3.2. Corollary. Every closed subset of $[\mathbf{N}]$ is completely Ramsey. 
The next lemma suggests the definition of completely Ramsey sets of Section 2.

3.3. Lemma. Let $\left\{X_{i}\right\}$ be an arbitrary sequence of subsets of $[\mathbf{N}]$. Let also a, $A$ be sets. We suppose that for every $L \subset A$,

$$
[a, L] \not \subset \bigcap_{i=1}^{\infty} X_{i} .
$$

Then there exist $b \subset A$ with $b>a, B \subset A$ and $i_{0}$ such that

$$
[a \cup b, L] \not \subset X_{i_{0}}
$$

for every $L \subset B$.

Proof. Assume the contrary. We choose $x_{1} \in A=A_{1}$ with $x_{1}>a$. Then the triple $\left(x_{1}, 1, A\right)$ does not satisfy $(*)$, so there exists $A_{2} \subset A$ with $\left[a \cup\left\{x_{1}\right\}, A_{2}\right] \subset X_{1}$. Suppose that natural numbers and sets

$$
\begin{gathered}
x_{1}<x_{2}<\cdots<x_{k}, \\
A=A_{1} \supset A_{2} \supset \cdots \supset A_{k} \supset A_{k+1}
\end{gathered}
$$

have been constructed such that for every $i \leq k, x_{i} \in A_{i}$ and for every nonempty $b \subset\left\{x_{1}, \ldots, x_{i}\right\}$ we have $\left[a \cup b, A_{i+1}\right] \subset X_{j}$ for all $j \leq i$. Then for every nonempty $b \subset\left\{x_{1}, \ldots, x_{k}\right\}$ we have $\left[a \cup b, A_{k+1}\right] \subset X_{j}$ for every $j \leq k$. Using a repeated argument we find $x_{k+1}$ and $A_{k+2}$ extending (**). The set $B=\left\{x_{i}: i=1,2, \ldots\right\}$ constructed in this way satisfies $[a, B] \subset \bigcap_{i=1}^{\infty} X_{i}$ which is a contradiction, and the proof is complete.

3.4. Corollary. If $\left\{R_{i}\right\}$ is a sequence of completely Ramsey sets, then the union $\bigcup_{i=1}^{\infty} R_{i}$ is a completely Ramsey set.

Proof. Let $a, A$ be sets. Let us assume that there does not exist $B \subset A$ with $[a, B] \subset \bigcup_{i=1}^{\infty} R_{i}$. Then by Lemma 3.1 there exists $B_{1} \subset A$ with $[a \cup b, L] \not \subset \bigcup_{i=1}^{\infty} R_{i}$ for every $b \subset B_{1}$ and every $L \subset B_{1}$. If we assume that there does not exist $B \subset B_{1}$ with $[a, B] \subset \bigcap_{i=1}^{\infty} R_{i}^{c}$, then by Lemma 3.3 there exist $b \in B_{1}$ with $b>a, B^{\prime} \subset B_{1}$ and $i_{0}$ such that $[a \cup b, L] \not \subset R_{i_{0}}^{c}$ for every $L \subset B^{\prime}$. Since $R_{i_{0}}$ is completely Ramsey, there exists $B_{2} \subset B^{\prime}$ with $\left[a \cup b, B_{2}\right] \subset R_{i_{0}} \subset \bigcup_{i=1}^{\infty}, R_{i}$, a contradiction.

From Corollaries 3.2 and 3.4 the next theorem follows.

3.5. Theorem (Galvin-Prikry). Every Borel subset of $[\mathbf{N}]$ is completely Ramsey.

Also from Lemma 3.3 we have the following lemma:

3.6. Lemma. If $\left\{R_{i}\right\}$ is a sequence of completely Ramsey sets and $[a, A] \subset \bigcup_{i=1}^{\infty} R_{i}$, then there exist $b \subset A$ with $b>a, B \subset A$ and $i_{0}$ such that $[a \cup b, B] \subset R_{i_{0}}$.

Finally the following lemma is an immediate consequence of Lemma 3.1.

3.7. Lemma. For every $X \subset[\mathbf{N}]$ the set $\widehat{X}$ is completely Ramsey.

We are now in a position to prove that analytic sets in $[\mathbf{N}]$ are completely Ramsey.

3.8. Theorem (Silver). Every analytic subset of $[\mathbf{N}]$ is completely Ramsey. 
Proof. Let $X \subset[\mathbf{N}]$ be analytic and let $a, A$ be sets. Also let $f: \mathcal{N} \rightarrow[\mathbf{N}]$ be a continuous function with $X=f(\mathcal{N})$. For every $s$ we set $X_{s}=f\left[\mathcal{N}_{s}\right]$. Certainly, $X_{\varnothing}=X$. Then for every $s$ we have

$$
X_{s}=\bigcup_{i=1}^{\infty} X_{\langle s, i\rangle} \quad \text { and } \quad X=\bigcup_{\sigma} \bigcap_{j} X_{\sigma \mid j}
$$

and since $f$ is continuous

$$
X=\bigcup_{\sigma} \bigcap_{j} \bar{X}_{\sigma \mid j}
$$

Since $X_{s} \subset \widehat{X}_{s} \subset \bar{X}_{s}$, we also have

$$
X=\bigcup_{\sigma} \bigcap_{j} \widehat{X}_{\sigma \mid j}
$$

By Lemma 3.7 each $\widehat{X}_{s}$ is completely Ramsey, so the set

$$
\bigcup_{s}\left(\widehat{X}_{s} \backslash \bigcup_{i=1}^{\infty} \widehat{X}_{\langle s, i\rangle}\right)
$$

is completely Ramsey. If we assume that there exists $B \subset A$ with

$$
[a, B] \subset \bigcup_{s}\left(\widehat{X}_{s} \backslash \bigcup_{i=1}^{\infty} \widehat{X}_{\langle s, i\rangle}\right),
$$

then, by Lemma 3.6, there exist $b \in B$ with $a<b, B^{\prime} \subset B$ and $s$ such that

$$
\left[a \cup b, B^{\prime}\right] \subset \widehat{X}_{s} \backslash \bigcup_{i=1}^{\infty} \widehat{X}_{\langle s, i\rangle}
$$

which is impossible because of (1) and the definition of $\widehat{X}_{s}$. Therefore there exists $B_{1} \subset A$ such that

$$
\left[a, B_{1}\right] \cap\left[\bigcup_{s}\left(\widehat{X}_{s} \backslash \bigcup_{i=1}^{\infty} \widehat{X}_{\langle s, i\rangle}\right)\right]=\varnothing .
$$

Let us assume that there does not exist $B \subset B_{1}$ with $[a, B] \subset\left(\widehat{X}_{\varnothing}\right)^{c}=(\widehat{X})^{c} \subset X^{c}$. Then there exists $B \subset B_{1}$ with $[a, B] \subset \widehat{X}_{\varnothing}$. We claim that $[a, B] \subset X$. Indeed, let $L \in[a, B]$. Then $L \in \widehat{X}_{\varnothing}$ and by (3) there exists $i_{1}$ with $L \in \widehat{X}_{\left\langle i_{1}\right\rangle}$. Repeating this argument we find $i_{2}$ with $L \in \widehat{X}_{\left\langle i_{1}, i_{2}\right\rangle}$. Continuing in this way we find a sequence $\sigma=\left\langle i_{1}, i_{2}, \ldots\right\rangle$ with $L \in \bigcap_{j} \widehat{X}_{\sigma \mid j} \subset X$, and the proof of the theorem is complete.

3.9. Remark. Ellentuck's topology on $[\mathbf{N}]$ is the topology with base the family of sets of the form $[a, B]$. The above lemmas and corollaries can be used to obtain easily the characterization of Ellentuck that a subset of $[\mathbf{N}]$ is completely Ramsey if and only if it has the Baire property in Ellentuck's topology. Also, for every $X$, the set $\widehat{X}$ is a minimal completely Ramsey cover of $X$ with respect to the $\sigma$-ideal (Lemma 3.6) of the Ramsey null sets, i.e. of the completely Ramsey sets $Y$ with the property $\forall[a, A]\left([a, A] \cap Y^{c} \neq \varnothing\right)$. 


\section{REFERENCES}

[1] T. J. Carlson, Some unifying principles in Ramsey theory, Discrete Math. 68 (1988), 117-169. MR 89b:04006

[2] T. J. Carlson and S. G. Simpson, A dual form of Ramsey's theorem, Adv. Math. 53 (1984), 265-290. MR 85h:04002

[3] E. Ellentuck, A new proof that analytic sets are Ramsey, J. Symbolic Logic 39 (1974), 163165. MR 50:1887

[4] F. Galvin and K. Prikry, Borel sets and Ramsey's theorem, J. Symbolic Logic 38 (1973), 193-198. MR 49:2399

[5] A. Kechris, Classical Descriptive Set Theory, Springer-Verlag, New York, 1995. MR 96e:03057

[6] J. Silver, Every analytic set is Ramsey, J. Symbolic Logic 35 (1970), 60-64. MR 48:10807

Department of Mathematics, University of Athens, Panepistemiopolis, Athens 15784, Greece 